\title{
ERRATUM
}

Y. Sakata $\cdot$ N. Kubo $\cdot$ M. Morishita $\cdot$ E. Kitadani

M. Sugiyama $\cdot$ M. Hirai

\section{QTL analysis of powdery mildew resistance in cucumber (Cucumis sativus L.)}

Published online: 6 January 2006

(C) Springer-Verlag 2006

\section{Theor Appl Genet (2005) 112:243-250}

The name of the fifth author, M. Sugiyama, was rendered incorrectly in the original publication.

The online version of the original article can be found at http:// dx.doi.org/10.1007/s00122-005-0121-1

Y. Sakata $\cdot$ M. Sugiyama

Department of Fruit Vegetables, National Institute of vegetables and Tea Science, Ano, Mie 514-2392, Japan

N. Kubo $\cdot$ M. Hirai $(\bowtie)$

Graduate School of Agriculture, Kyoto Prefectural University,

Kyoto Prefectural Institute of Agricultural Biotechnology,

74 Oji, Kitaihayazuma, Seika, Kyoto 619-0244, Japan

E-mail: mhirai@kab.seika.kyoto.jp

Tel.: + 81-774-933526

Fax: $+81-774-933528$

M. Morishita

Department of Crop Breeding, National Agricultural Research Center for Hokkaido Region, Sapporo 062-0045, Japan

E. Kitadani

Department of vegetable and Flower Research,

National Agricultural Research Center for Kyushu Okinawa

Region, Kurume 839-8503, Japan 